\title{
Concentraciones sanguíneas de sulfadoxina y pirimetamina según la respuesta terapéutica antimalárica, en dos municipios de Antioquia, Colombia
}

\author{
Jaime Carmona, ${ }^{1}$ Adriana Pabón, ${ }^{1}$ Diana Márquez, ${ }^{2}$ \\ Carlos López, ${ }^{3}$ Gladys Morales ${ }^{4}$ y Silvia Blair ${ }^{1}$
}

Forma de citar Carmona J, Pabón A, Márquez D, López C, Morales G, Blair S. Concentraciones sanguíneas de sulfadoxina y pirimetamina según la respuesta terapéutica antimalárica, en dos municipios de Antioquia, Colombia. Rev Panam Salud Publica. 2005;18(2):75-83.

RESUMEN Problema. Se ha observado un aumento constante del índice de fracaso terapéutico de la combinación sulfadoxina-pirimetamina (SDX-PIR) en el tratamiento de la malaria por Plasmodium falciparum sin complicaciones.

Objetivo. Cuantificar, mediante cromatografía de líquidos de alta resolución (HPLC), las concentraciones sanguíneas de SDX-PIR en pacientes con buena respuesta clínica y sin respuesta al tratamiento.

Métodos. En 2002 se llevó a cabo un estudio experimental con asignación aleatoria y sin anonimato para evaluar el tratamiento con la combinación SDX-PIR en una población de 79 pacientes de dos municipios del departamento de Antioquia en Colombia (Turbo: 45; Zaragoza: 34), de uno y otro sexo y de 1 a 60 años de edad, con malaria por Plasmodium falciparum sin complicaciones

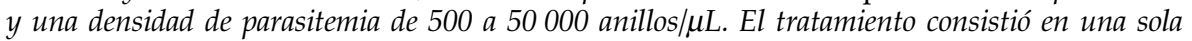
dosis, administrada bajo supervisión médica, de SDX $(25 \mathrm{mg} / \mathrm{kg})$ y PIR $(1,25 \mathrm{mg} / \mathrm{kg})$ combinadas en comprimidos (500 mg y $25 \mathrm{mg}$ de SDX y PIR, respectivamente) y se realizó seguimiento clínico y parasitológico por 21 días. Las concentraciones de SDX y PIR se midieron dos horas después de la administración del medicamento y el día del fracaso terapéutico en los casos en que se produjo. Resultados. A las 2 horas de haberse administrado el medicamento la concentración sanguínea mediana de SDX fue de 136,6 $\mathrm{mol} / \mathrm{L}$ en los pacientes que mostraron respuesta clínica adecuada y de 103,4 $\mu \mathrm{mol} / \mathrm{L}$ en quienes no respondieron al tratamiento $(\mathrm{P}=0,13)$. La mediana de PIR fue 848,4 y 786,1 nmol/L en pacientes con respuesta clínica adecuada y fracaso terapéutico, respectivamente $(\mathrm{P}=0,40)$. Las concentraciones tampoco mostraron diferencia significativa entre los casos de fracaso temprano y tardío. La correlación lineal entre las concentraciones de SDX y PIR fue cercana a cero $(\mathrm{r}=0,13)$.

Discusión y conclusiones. Con respecto a 1998, el fracaso del tratamiento con la combinación SDX-PIR aumentó de $13 \%$ a $22 \%$ en Turbo y de $9 \%$ a $26 \%$ en Zaragoza. La falta de respuesta en 2002 no pudo explicarse por concentraciones (menores) de los medicamentos en sangre.

Palabras clave Malaria falciparum, antimaláricos, sulfadoxina, pirimetamina, cromatografía líquida de alta presión.

1 Grupo Malaria, Universidad de Antioquia, Medellín, Colombia. Toda correspondencia deberá dirigirse a: Silvia Blair, Calle 62 \# 52-59, Torre 1, Piso 6. Laboratorio 610, Sede de Investigación Universitaria (SIU), Universidad de Antioquia, Medellín,
Colombia. Fax: 57 (4) 2106487. Correo electrónico: sblair@catios.udea.edu.co

2 Facultad de Química Farmacéutica, Universidad de Antioquia, Medellín, Colombia.
3 Facultad de Ciencias Exactas y Naturales, Universidad de Antioquia, Medellín, Colombia.

4 Universidad de Medellín, Colombia. 
La combinación de sulfadoxina (N1-[5,6-dimetoxi-4-pirimidinil]sulfanilamida) con pirimetamina $(2,4-$ diamino-5[5-clorofenil]-6-etilpiridina) se emplea para el tratamiento de la malaria por Plasmodium falciparum sin complicaciones. Su acción se explica por el bloqueo sucesivo de dos enzimas plasmodiales que participan en la biosíntesis del ácido fólico. Específicamente, su condición de análogos estructurales y antagonistas competitivos del ácido paraaminobenzoico (PABA) les da la capacidad de impedir que el parásito utilice el PABA en la síntesis de ácido fólico. La sulfadoxina es un inhibidor competitivo de la enzima dihidropteroato sintetasa, que incorpora el PABA en el ácido dihidropteroico para formar un compuesto que con participación del glutamato forma el ácido dihidrofólico. Este, por acción de la enzima dihidrofolato reductasa, se reduce a ácido tetrahidrofólico. Esta última enzima es inhibida por la pirimetamina (1-3).

La sulfadoxina (SDX) y la pirimetamina (PIR) se combinan a razón de 20 a 1 en los comprimidos formulados para el tratamiento antimalárico; la SDX tiene actividad antimalárica contra las cuatro especies de plasmodios que afectan al ser humano. Sin embargo, en algunas zonas endémicas, especialmente en el Sudeste Asiático y en América del Sur, existen cepas de $P$. falciparum resistentes a esta combinación (4-14). Estudios realizados en Colombia para evaluar su eficacia terapéutica contra $P$. falciparum mostraron fracaso terapéutico en proporciones de 9 a $13 \%$ en el departamento de Antioquia y de 3 a $6 \%$ en la costa del Pacífico, entre los años 1997 y 1999 (15-21). Tales resultados son preocupantes, porque la cloroquina (CQ), fármaco de primera línea en los esquemas de tratamiento antimalárico, mostró fracaso terapéutico en 67 a 97\% de los casos en 1998 (15-18, 22-25).

El presente estudio da cuenta de la estimación de la eficacia terapéutica y las concentraciones sanguíneas de sulfadoxina y pirimetamina el día del ingreso al estudio (o día cero) y el día del fracaso (cuando se presentó), en 79 pacientes con malaria por $P$. falciparum residentes de los dos municipios con malaria endémica mencionados. En el período de 1996 a 2000, el municipio de Turbo, situado en el Urabá antioqueño, presentaba un índice parasitario anual (IPA) promedio de 40 por 1000 habitantes; para el 2001 registraba 2203 casos por P. vivax y 1094 por $P$. falciparum. En ese mismo periodo (1996-2000), el otro municipio, Zaragoza, situado en el Bajo Cauca antioqueño, presentaba un IPA promedio de 156 por 1000 habitantes; para 2001 registraba 306 casos por $P$. vivax y 160 casos por $P$. falciparum (26).

\section{MATERIALES Y MÉTODOS}

\section{Tipo de estudio}

En 2002 se realizó un estudio experimental en el que cada paciente con malaria por $P$. falciparum sin complicaciones se sometió a tratamiento con uno de tres esquemas (CQ, SDX-PIR, $\mathrm{CQ}+$ SDX-PIR), asignado de forma aleatoria. El diseño no fue ciego, ya que el médico que asignó el tratamiento fue el mismo que evaluó la respuesta terapéutica.

\section{Población de referencia}

La conforman los pacientes con malaria por P. falciparum sin complicaciones y ambulatorios, de uno $\mathrm{u}$ otro sexo y de 1 ó más años de edad, residentes en los municipios mencionados (en la zona urbana o en la rural) que consultaron por propia iniciativa a algún puesto de diagnóstico de malaria. Estos puestos, administrados por la dirección local de salud de cada municipio, con el apoyo financiero de la Dirección Seccional de Salud de Antioquia, realizan labores de diagnóstico, tratamiento y prevención de las enfermedades trasmitidas por vectores. Dado que en todos los municipios la atención de la malaria es financiada por el Estado, la presente investigación se acogió a este beneficio, además de obtener cobertura económica completa de la atención hospitalaria de los pacientes que la requirieran, ya fuera por complicaciones de la enfermedad o por el tratamiento recibido.

\section{Diseño y cálculo de la muestra}

Para calcular el tamaño de la muestra se aplicó la fórmula:

$$
n=\frac{N \times Z^{2} \times p(1-p)}{\left[N \times e^{2}\right]+\left[Z^{2} \times p(1-p)\right]}
$$

donde:

$n=$ tamaño muestral calculado

$N=$ población de pacientes con malaria por P. falciparum, que consultan al puesto de malaria

$Z \quad=$ nivel de confianza, que se fija en $95 \%$, lo que supone un error alfa (nivel de significación) de $5 \%$

$p=$ proporción esperada de ocurrencia del fracaso terapéutico

$1-p=$ complemento de $p$

$e \quad=$ error de muestreo, definido aquí en $12 \%$.

Para calcular el tamaño de la muestra de cada municipio (Turbo y Zaragoza) se empleó la información sobre frecuencia de malaria por P. falciparum correspondiente a 1999 (27), los datos conocidos acera de la frecuencia de fracaso terapéutico en los dos lugares $(15,17)$ y los valores de $Z$ y de $e$ ya indicados. De esa manera, se completó la siguiente información:

\begin{tabular}{ccccccc}
\hline$N$ & $Z$ & $p$ & $1-p$ & $e$ & $n$ & $\begin{array}{c}\text { Medicamento } \\
\text { y lugar }\end{array}$ \\
\hline 595 & 1,96 & 0,13 & 0,87 & $12 \%$ & 29 & $\begin{array}{c}\text { SDX-PIR } \\
\text { en Turbo }\end{array}$ \\
466 & 1,96 & 0,09 & 0,91 & $12 \%$ & 21 & $\begin{array}{c}\text { SDX-PIR } \\
\text { en Zaragoza }\end{array}$ \\
\hline
\end{tabular}

Según lo anterior, el tamaño de las muestras de Turbo y Zaragoza fue de 21 y 29, respectivamente. Al final, se incluyeron 45 pacientes en Turbo y 34 en Zaragoza.

\section{Aspectos éticos}

A cada paciente con malaria por $P$. falciparum se le informó del estudio, sus objetivos, riesgos y beneficios. Una 
vez aceptada su participación (o la de su hijo) y cumplidos los criterios de inclusión, se obtuvo la firma de una carta de consentimiento fundamentado que cumplía con los requisitos establecidos por el Comité de Ética de la Universidad de Antioquia.

\section{Criterios de inclusión, exclusión y retiro}

Se incluyeron todos los pacientes con malaria que satisfacían los criterios del protocolo estándar de evaluación de la respuesta terapéutica a los antimaláricos de la OMS/OPS de 1998 $(28,29)$ y que además tuvieran facilidad de regresar a las evaluaciones de seguimiento.

\section{Diagnóstico parasitológico}

El diagnóstico de malaria se realizó mediante examen de gota gruesa, que se efectuó con objetivo de $100 \times$ (aumento de 1000 veces), y el diagnóstico se basó en el estudio de 200 campos microscópicos. La parasitemia se calculó con base en 200 leucocitos y un estándar de 8000 leucocitos $/ \mu \mathrm{L}$ y se expresó en anillos/ $\mu \mathrm{L}$ (30). El examen de gota gruesa se consideró negativo cuando no se detectó ninguna forma asexual en 200 campos microscópicos.

\section{Tratamiento antimalárico}

Se usaron comprimidos de $500 \mathrm{mg}$ de SDX y $25 \mathrm{mg}$ de PIR producidos por Laboratorios Eron, S. A., de La Habana, Cuba, y suministrados al estudio por el Ministerio de Salud de Colombia por conducto de la Dirección Seccional de Salud de Antioquia. El producto suministrado a los pacientes llevó la clave de identificación de lote "sul 50600606"; la gestión y la conservación de los certificados de calidad de los medicamentos son funciones del Ministerio de Protección Social de Colombia. La SDX-PIR se administró a razón de $25 \mathrm{mg} / \mathrm{kg}$ de SDX por 1,25 mg/kg de PIR y se suministró en una sola toma, todo conforme a las disposiciones del Ministerio de Salud de Colombia; el investigador administró el medicamento a cada paciente, acompañado con agua. El tratamiento se supervisó durante la primera media hora; en caso de vómito se repitió la dosis completa. Si el paciente vomitaba nuevamente se retiraba del estudio y se remitía al hospital municipal para atención hospitalaria. El último día de seguimiento (día 21) se administró a todos los pacientes primaquina $(0,75 \mathrm{mg} / \mathrm{kg}$ en dosis única). El tratamiento de rescate en los sujetos que presentaron fracaso terapéutico consistió en sulfato de quinina (comprimidos de $300 \mathrm{mg}, 10 \mathrm{mg} /$ $\mathrm{Kg}$ cada 8 horas), más clindamicina (cápsulas de 300 mg, 5 mg/Kg) cada 6 horas durante tres y cinco días, respectivamente, como lo indica el esquema oficial de tratamiento de la malaria del Ministerio de Protección Social de Colombia.

\section{Evaluación in vivo de la respuesta terapéutica}

El día del ingreso (día 0) se obtuvo una muestra de sangre para examen de gota gruesa y frotis de sangre periférica; luego se suministró el tratamiento correspondiente. Para evaluar la eficacia terapéutica de los antimaláricos se aplicó el protocolo estándar de la Organización Panamericana de la Salud (OPS) 98 (28, 29). La evaluación se realizó mediante seguimiento clínico durante un período de 21 días, que fue menor en los pacientes que no respondieron al tratamiento. Se efectuaron visitas de control clínico y parasitológico al cabo de 1, 2, 3, 7, 14 y 21 días de haberse iniciado el tratamiento, para evaluar la eventual aparición de signos o síntomas de enfermedad malárica activa o de complicación de la enfermedad.

\section{Clasificación de la respuesta terapéutica}

La respuesta se clasificó de acuerdo con el protocolo OPS 98 (29), que evalúa la respuesta clínica y parasitológica de la siguiente manera:
Fracaso terapéutico precoz (FTP):

- Signos de peligro o malaria grave los días 1, 2 ó 3.

- Parasitemia al día 2 mayor que al día 0.

- Parasitemia al día 3 igual o mayor de $25 \%$ del recuento del día 0.

Fracaso terapéutico tardío (FTT):

- Signos de peligro o malaria grave después del día 3, junto con parasitemia.

- Regreso del paciente entre los días 4 y 21, por deterioro clínico aunado a parasitemia.

- Presencia de parasitemia por P. falciparum en los días 7, 14 ó 21.

Respuesta clínica adecuada (RCA):

- El paciente no presenta ningún criterio de fracaso terapéutico temprano o tardío y el examen de gota gruesa se mantiene negativo entre el día 4 y la última visita de control, el día 21.

\section{Procesamiento de la muestra de sangre para medir la concentración de sulfadoxina y pirimetamina}

Para medir la concentración sanguínea de SDX y de PIR, a las dos horas postratamiento y en el momento del fracaso (cuando se presentó) se realizó punción capilar en el dedo de la mano y con ayuda de una micropipeta Gilson ${ }^{\circledR}$ se extrajeron con precisión $100 \mu \mathrm{L}$ de sangre. La muestra se depositó en papel de filtro Whatman del número 3 y se dejó secar a temperatura ambiental por 30 minutos, sin exposición a la luz; después, el papel con la muestra se introdujo en bolsas de plástico resellables y se guardó a $4{ }^{\circ} \mathrm{C}$. La muestra se obtuvo por triplicado.

Para medir la concentración sanguínea de SDX y PIR en el papel de filtro se empleó el método propuesto por Green et al. (31), que utiliza cromatografía de líquidos de alta resolución (high-resolution liquid chromatography, HPLC) con columna en fase inversa y detector de luz ultravioleta (UV).

Luego, el papel de filtro con la muestra se cortó en pequeños trozos, que se introdujeron en un tubo de polipropileno para microcentrífuga (tubo de Eppendorf) de $2 \mathrm{~mL}$. Luego se añadieron $250 \mathrm{~mL}$ de ácido clorhídrico $(0,1 \mathrm{M})$ y 
$750 \mathrm{~mL}$ de acetonitrilo. Las muestras se centrifugaron a 14000 revoluciones por minuto durante 15 minutos y, una vez separada la fase orgánica, se les añadió $1 \mathrm{~mL}$ de tampón (amortiguador) fosfato de potasio a un $\mathrm{pH}$ de 5,6 y se sometieron por 2 minutos a ultrasonido. Después de este tratamiento se añadieron 2,5 $\mathrm{mL}$ de cloroformo y la preparación se mezcló en un sistema de remolino (vortex), se dejó en reposo y la fase clorofórmica se separó por evaporación. El residuo seco se disolvió con $1 \mathrm{~mL}$ de fase móvil, se filtró con membrana de nailon con poro de 0,45 $\mu \mathrm{m}$ de diámetro y se inyectaron $20 \mu \mathrm{L}$ en el cromatógrafo de líquidos con una columna de HPLC LiChroCART ${ }^{\circledR} 125-4$ con cartucho LiChrospher ${ }^{\circledR}$ de 100 RP$18(5 \mu \mathrm{m})$, provista de guardacolumnas LiChroCART $^{\circledR} 4-4^{\circledR}$ de 100 RP-18 (5 $\mu \mathrm{m})$. Como fase móvil se utilizó una mezcla 50:50 de acetonitrilo y fosfato de potasio, que se hizo correr a una velocidad de $0,7 \mathrm{~mL} / \mathrm{min}$, con longitud de onda de $272 \mathrm{~nm}$ durante 0 a 3 minutos y de $287 \mathrm{~nm}$ por 3 a 8 minutos.

Para calcular la concentración de SDX y de PIR en las muestras de cada paciente se comparó el área obtenida con el patrón (estándar) (SDX y PIR) después de haber establecido una curva de calibración con análisis de regresión (modelo lineal: $Y=a+b X$ ). En el caso de la sulfadoxina, el área fue igual a 715,508 + 97,762 $\times$ concentración, el coeficiente de correlación de 0,996, $r^{2}$ de 99,147 y el error estándar de la estimación de 1 019,63. En el de la pirimetamina, el área fue igual a 1,010 + 0,066 × concentración, el coeficiente de correlación de 0,997 y el error estándar de la estimación de 0,533.

\section{Análisis estadístico}

Se ingresaron los datos en una base construida con el programa Epilnfo 6,04 , con el cual se validaron y procesaron. Se calcularon medidas estadísticas de resumen de tendencia central, dispersión, asimetría y curtosis. Los gráficos se construyeron con Statgraphics Plus 7.1. Como prueba de ajuste de los datos a la distribución normal se usó la de Kolmogorov-Smirnov, conte- nida en el programa Statgraphics Plus 7.1. Mediante una prueba de ji al cuadrado se evaluó la asociación entre la respuesta terapéutica (respuesta clínica adecuada, fracaso temprano, fracaso tardío) y el nivel endémico de malaria (alto y bajo) de cada municipio (Turbo y Zaragoza).

Con la prueba de Kruskal y Wallis (K-W) se compararon las medianas de las concentraciones de cada fármaco (SDX y PIR) en cada grupo de respuesta terapéutica, tanto a las 2 horas de administrado el medicamento SDXPIR como en el momento de detectar el fracaso terapéutico.

Con la prueba de Wilcoxon para mediciones repetidas se compararon las medianas de las concentraciones de cada medicamento los días cero y de fracaso del tratamiento (cuando lo hubo). Siempre se consideró significativo un valor $P$ menor de 5\% $(P<0,05)$.

\section{RESULTADOS}

Se evaluó la eficacia terapéutica de la combinación SDX-PIR en 79 pacientes con malaria por $P$. falciparum, 45 en el municipio de Turbo y 34 en el de Zaragoza. Las causas de exclusión y de retiro se muestran en el cuadro 1 . El municipio de Turbo presentó un índice de fracaso terapéutico de $22 \%$ (10/45), con igual proporción de FTP y FTT, 11\% $(n=5)$. El municipio de Zaragoza tuvo $26 \%(9 / 34)$ de fracaso con la SDX, 21\% $(n=7)$ de forma precoz y $6 \%(n=2)$ de forma tardía. De 79 personas en quienes se evaluó la respuesta terapéutica, en 76 se logró realizar la medición de las concentraciones sanguíneas de SDX y de PIR a las dos horas de administrado el tratamiento; de ellas, 42 pertenecían a Turbo y 34 a Zaragoza. En 15 pacientes del total de 18 que tuvieron fracaso terapéutico la concentración del medicamento se midió también en el momento de registrarse este.

La prueba de ajuste de los datos a la distribución normal indica que la distribución de SDX puede considerarse estadísticamente normal. Con respecto a la PIR, 15 pacientes presentan un valor inferior al límite de detección (5,99 nmol/L), lo que apunta a una distribución bimodal; no obstante, al suprimirlos del análisis, la curva se torna normal (figuras 1 a 4). En el cuadro 2 se observa que, en el caso de la SDX, los 76 valores presentan un promedio aritmético $\mathrm{y}$ una mediana iguales (129 $\mu \mathrm{mol} / \mathrm{L})$, mientras que la moda es un poco menor $(105,1 \mu \mathrm{mol} / \mathrm{L})$. La tendencia a la dispersión es relativamente escasa, a juzgar por los valores de la desviación estándar $(64,5 \mu \mathrm{mol} / \mathrm{L})$ y particularmente del coeficiente de variación $(50 \%)$. La asimetría y la curtosis son casi nulas. De

CUADRO 1. Causas de exclusión y retiro de los pacientes con malaria. Turbo y Zaragoza, Antioquia, Colombia, 2002

\begin{tabular}{|c|c|c|}
\hline \multirow[b]{2}{*}{ Causa } & \multicolumn{2}{|c|}{ Número de pacientes/ municipio } \\
\hline & Turbo & Zaragoza \\
\hline Criterio de exclusión & - & 1 \\
\hline Antecedentes de alergias a medicamentos antimaláricos & 4 & 1 \\
\hline No vivir en la zona de estudio & - & 4 \\
\hline Vómito persistente (> dos veces en las últimas 24 horas) & - & 2 \\
\hline Haber recibido antibióticos en la última semana & 1 & 1 \\
\hline Coluria, oliguria, anuria o deshidratación intensa & Plasmodium vivax (4) & Plasmodium \\
\hline $\begin{array}{l}\text { Presencia de enfermedad concomitante que interfiriera } \\
\text { con la evolución clínica del cuadro de malaria }\end{array}$ & $\begin{array}{c}\text { Dengue (1) } \\
\text { Infección respiratoria (1) }\end{array}$ & $\operatorname{vivax}(4)$ \\
\hline Recuento de parásitos inferior a 500 anillos $/ \mu \mathrm{L}$ & 1 & 1 \\
\hline Presencia de esquizontes & 1 & - \\
\hline \multicolumn{3}{|l|}{ Criterio de retiro } \\
\hline $\begin{array}{l}\text { Inasistencia a las consultas de control en algún día del } \\
\text { tratamiento antimalárico }\end{array}$ & 6 & 2 \\
\hline Inasitencia a 3 consultas de control durante el seguimiento. & - & 5 \\
\hline Total & 19 & 21 \\
\hline
\end{tabular}


la misma forma la PIR tiene un promedio aritmético y una mediana casi iguales $(844,0 \mathrm{nmol} / \mathrm{L}$ y $830,3 \mathrm{nmol} / \mathrm{L})$. La moda fue de 184,2 nmol/L al suprimir los 15 valores que estuvieron debajo del límite de detección. La dispersión es moderada (desviación estándar de 354,2 nmol/L y coeficiente de variación de $41,9 \%$ ). La asimetría es escasa y la curtosis está dentro del intervalo normal.

En el cuadro 3 se resumen los valores estadísticos de las concentraciones sanguíneas de SDX y PIR por respuesta terapéutica. Los pacientes con respuesta clínica adecuada presentaron una mediana de $136,6 \mu \mathrm{mol} / \mathrm{L}$ para SDX, en comparación con 103,4 $\mu \mathrm{mol} / \mathrm{L}$ en los pacientes en quienes el tratamiento fracasó $(P=0,130)$. La mediana de las concentraciones sanguíneas de PIR de los pacientes que tuvieron respuesta adecuada fue de $848,4 \mathrm{nmol} / \mathrm{L}$, en comparación con 786,1 nmol/L en los que tuvieron fracaso $(P=0,402)$. Con ninguno de los dos medicamentos se advierte una diferencia significativa y tampoco se aprecia al comparar las medianas de las concentraciones sanguíneas de SDX y PIR según el tipo de respuesta terapéutica en tres grupos (respuesta clínica adecuada, fracaso temprano y fracaso tardío; $P=0,105$ con SDX y $P=$ 0,308 con PIR).

En el cuadro 4 se encuentran las concentraciones sanguíneas de SDX y PIR de los días cero y de fracaso terapéutico en las personas que no respondieron. En el fracaso temprano, las medianas de las concentraciones de SDX son prácticamente iguales los días cero y de fracaso. La concentración de PIR observada el día del fracaso es $14 \%$ menor que la del día cero, pero sin diferencia estadísticamente significativa. En el fracaso tardío, la concentración de SDX del día del fracaso es $64 \%$ más baja que la del día cero $(\mathrm{p}=0,063)$. La concentración de PIR fue $35 \%$ más baja el día del fracaso que el día cero. De las 15 personas en quienes se evaluó la concentración de los dos medicamentos, cuatro de ellas presentaron valores inferiores al límite de detección $(5,99 \mathrm{nmol} / \mathrm{L})$, uno con fracaso temprano y tres con fracaso tardío.

Entre las 18 personas que tuvieron fracaso terapéutico, este fue temprano en 11: dos pacientes el primer día, tres el
FIGURA 1. Distribución de la concentración de sulfadoxina en sangre. (DE, desviación estándar). Turbo y Zaragoza, Antioquia, Colombia, 2002

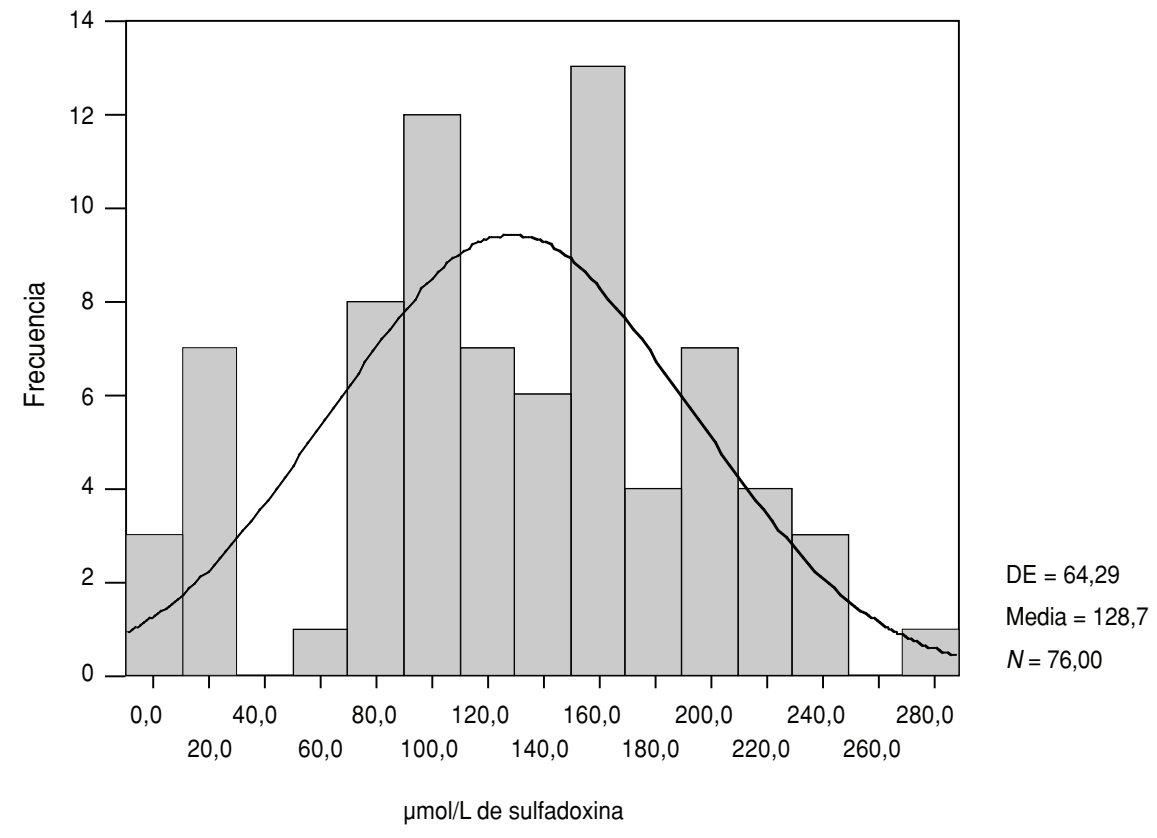

FIGURA 2. Sulfadoxina en sangre según la respuesta terapéutica. (RCA, respuesta clínica adecuada; FTP, fracaso terapéutico precoz; FTT, fracaso terapéutico tardío). Turbo y Zaragoza, Antioquia, Colombia, 2002

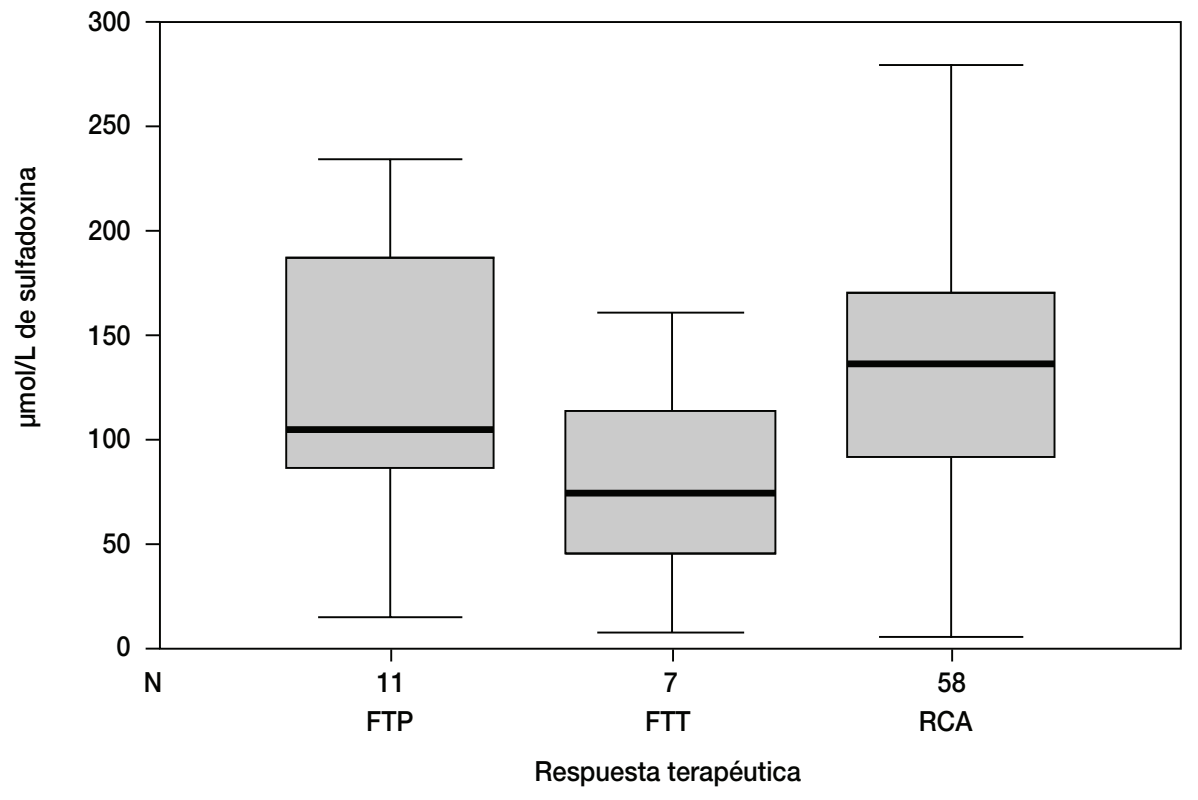

segundo y seis (55\%) el tercer día de seguimiento, y tardío en siete: uno el día 7, dos el día 14 y cuatro (57\%) el día 21.

Cuando la respuesta terapéutica a la SDX-PIR se analizó en función de otras variables, como el municipio, la zona de residencia (cabecera o periferia), la edad, el sexo y el grupo étnico, tampoco se encontraron diferencias estadísticamente significativas. 
FIGURA 3. Distribución de la concentración de pirimetamina en sangre. (DE, desviación estándar). Turbo y Zaragoza, Antioquia, Colombia, 2002

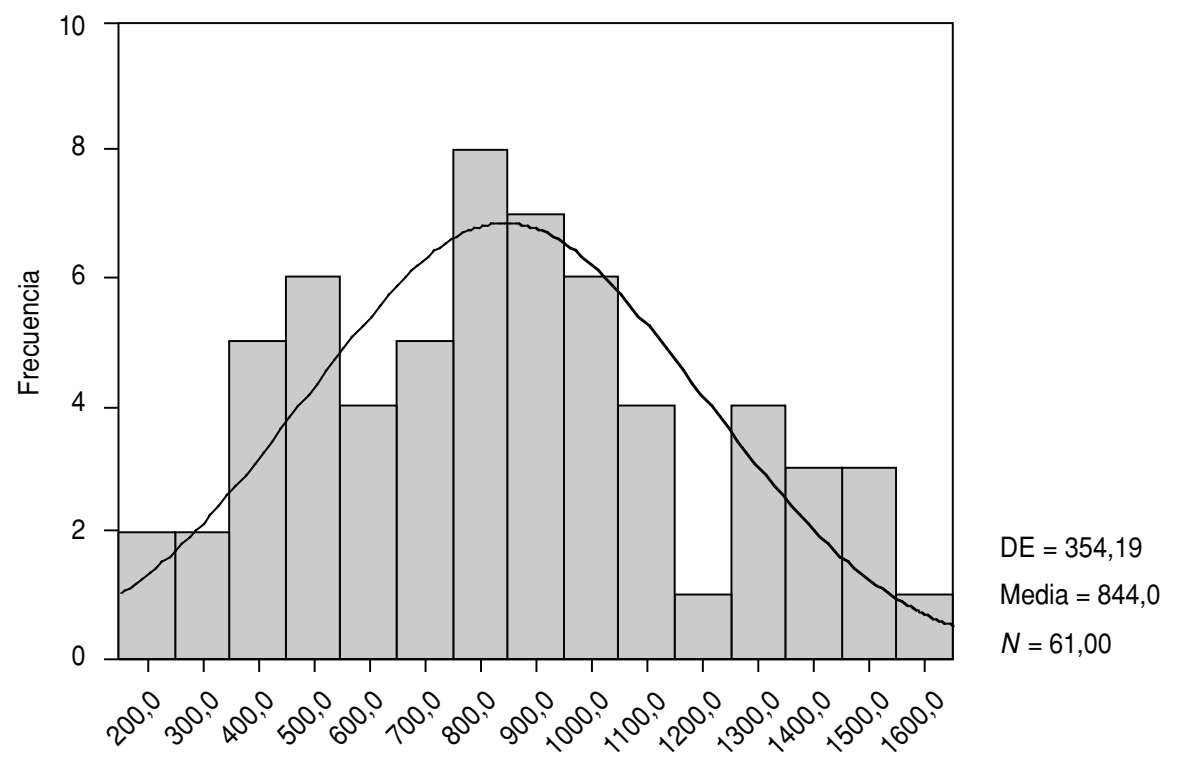

$\mathrm{nmol} / \mathrm{L}$ de pirimetamina

FIGURA 4. Pirimetamina en sangre según respuesta terapéutica (FTP, fracaso terapéutico precoz; FTT, fracaso terapéutico tardío; RCA, respuesta clínica adecuada). Turbo y Zaragoza, Antioquia, Colombia, 2002

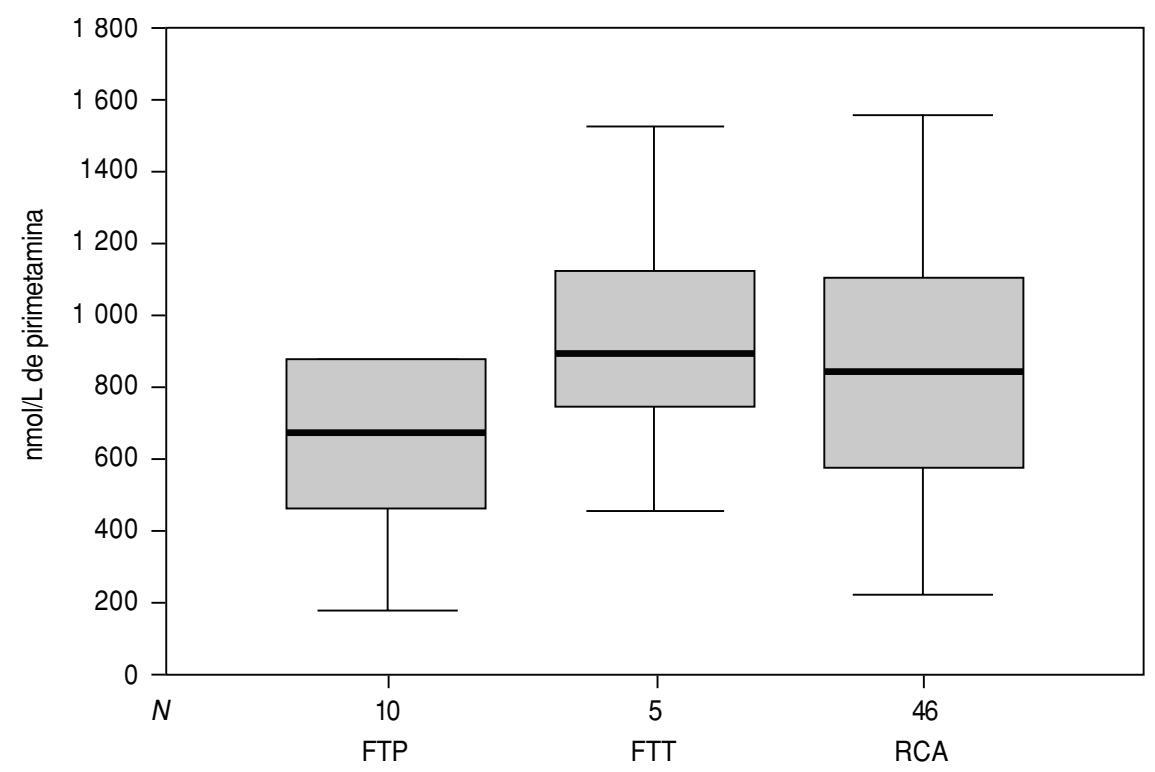

Respuesta terapéutica

\section{DISCUSIÓN}

La frecuencia del fracaso terapéutico con SDX-PIR (22\% en Turbo y $26 \%$ en Zaragoza) es alta y alcanza el límite de
25\% adoptado por la OPS para determinar la interrupción del uso del medicamento. Este hecho se suma a la falla de la cloroquina, que supera un índice de 90\% en ambos lugares, hecho que mo- tivó el retiro del esquema para el tratamiento de la malaria por $P$. falciparum en el departamento de Antioquia.

En ambos municipios estudiados el fracaso terapéutico de SDX-PIR no se explica por deficiencia de la concentración sanguínea del medicamento, como lo demuestra la observación, a las dos horas de su administración, de concentraciones sanguíneas estadísticamente similares en los grupos de buena respuesta terapéutica y de fracaso. Tales concentraciones, por otra parte, parecen adecuadas para el tratamiento de la malaria sin complicaciones originada por $P$. falciparum, según se deduce de dos argumentos:

a) tales concentraciones se produjeron después de una dosis estándar de SDX-PIR (25 y 1,25 mg $/ \mathrm{kg}$, respectivamente), recomendada por la OPS (protocolo de 1998) y que permite lograr una curación clínica y parasitológica en $90 \%$ de los enfermos cuando el parásito es sensible (no resistente), tal como lo hallamos en 1998 en Turbo y Zaragoza, donde hubo una respuesta terapéutica adecuada en $91 \%$ y $87 \%$ de los pacientes $(17,18)$. Se deduce, entonces, que la resistencia de $P$. falciparum a SDX-PIR ha aumentado en esos dos lugares;

b) las concentraciones sanguíneas observadas de SDX $(129 \mu \mathrm{mol} / \mathrm{L})$ y PIR (830,3 nmol/L), medidas en los pacientes con malaria dos horas después de administrado el medicamento, están cercanas a las concentraciones máximas (cmáx) señaladas por otros autores en personas sanas $(203,7 \pm 34,8 \mu \mathrm{mol} / \mathrm{L}$ para SDX y $860,5 \pm 261,4 \mathrm{nmol} / \mathrm{L}$ para PIR), valores alcanzados en tiempos máximos (tmáx) de 3,7 $\pm 1,7$ horas y 4,2 $\pm 2,7$ horas, respectivamente, usando una dosis única de $500 \mathrm{mg}$ de SDX y $25 \mathrm{mg}$ de PIR (32). Estas concentraciones observadas por los autores antes de los tmáx indican que la absorción de los fármacos ocurrió sin dificultad y que las concentraciones serían adecuadas.

No conocemos cuáles son las concentraciones parasiticidas mínimas in vivo de 
CUADRO 2. Valores estadísticos de las concentraciones sanguíneas de sulfadoxina (SDX) ( $\mu \mathrm{molg} / \mathrm{L})$ y de pirimetamina (PIR) (nmol/L)

\begin{tabular}{|c|c|c|c|c|}
\hline \multirow[b]{2}{*}{ Variable } & \multicolumn{2}{|c|}{ Día 0 (2 horas) } & \multicolumn{2}{|c|}{ Día de falla } \\
\hline & SDX & PIR & SDX & PIR \\
\hline \multicolumn{5}{|l|}{ Medidas de tendencia central } \\
\hline Tamaño de la muestra & 76,0 & 61 & 15,0 & 11,0 \\
\hline Promedio & 128,6 & 844,0 & 78,0 & 630,1 \\
\hline Mediana & 129,9 & 830,3 & 45,8 & 487,3 \\
\hline Moda & 105,1 & 184,2 & 121,5 & 225,2 \\
\hline \multicolumn{5}{|l|}{ Medidas de dispersión } \\
\hline Varianza & 4133,6 & 125448,0 & 3556,5 & 1800191,7 \\
\hline Desviación estándar & 64,5 & 354,2 & 59,6 & 431,8 \\
\hline Valor mínimo & 6,4 & 184,2 & 3,9 & 225,2 \\
\hline Valor máximo & 278,8 & 1554,9 & 176,6 & 1623,6 \\
\hline Recorrido & 272,3 & 1370,7 & 172,7 & 1398,5 \\
\hline Cuartil 1 & 88,3 & 558,5 & 28,4 & 295,1 \\
\hline Cuartil 2 & 129,9 & 830,3 & 45,8 & 487,3 \\
\hline Cuartil 3 & 170,2 & 991,2 & 137,3 & 450,7 \\
\hline Intervalo intercuartílico & 81,9 & 525,1 & 108,9 & 557,7 \\
\hline Coeficiente de variación & 50,13 & 41,9 & 76,45 & 68,5 \\
\hline \multicolumn{5}{|c|}{ Medidas de asimetría y curtosis } \\
\hline Asimetría & $-0,1$ & 0,245 & 0,3 & 1,45 \\
\hline Curtosis & $-0,5$ & $-0,711$ & $-1,6$ & 1,76 \\
\hline
\end{tabular}

SDX y PIR, lo que impide comparar con ellas nuestros datos. Entendemos que concentraciones como las informadas por Weidekamm et al. en 1982 (32) son parasiticidas y que las observadas en nuestros pacientes también lo son, según se deduce del hecho de que la mayoría se curaron y de que muy probablemente la falta de cura depende más de la resistencia parasitaria que de concentraciones sanguíneas insuficientes.

Se ha observado que se consiguen concentraciones parasiticidas de SDX-
PIR con dosis orales únicas de apenas 2,14 y $0,11 \mathrm{mg} / \mathrm{kg}$, respectivamente, las cuales curaron a $69 \%$ de los niños enfermos en Gabón (33). En Tanzania, en escolares asintomáticos con parasitemia por $P$. falciparum tratados con $100 \%, 50 \%$ y $25 \%$ de la dosis estándar de SDX-PIR, seguidos por 28 a 42 días, se logró la eliminación de los parásitos en la totalidad de los casos y en los tres grupos (34), lo que demostraría una alta eficacia antiplasmodial de la combinación SDX-PIR, aun en dosis muy bajas, como 6,25 y $0,312 \mathrm{mg} / \mathrm{kg}$, que es una cuarta parte de la dosis estándar. En 10 de estos niños, tratados con la dosis estándar de SDX-PIR (25 y 1,25 $\mathrm{mg} / \mathrm{kg}$ ), se midieron las concentraciones de SDX en sangre y plasma. La cantidad resultante fue mayor en plasma (razón sangre:plasma $=0,72$ ) y la mediana en sangre, de $303 \mu \mathrm{mol} / \mathrm{L}$, el primer día postratamiento. Los mismos autores informaron concentraciones inhibidoras mínimas (CIM) in vitro de SDX y PIR, en el caso de P. falciparum, de $5 \mu \mathrm{mol} / \mathrm{L}$ y $62 \mathrm{nmol} / \mathrm{L}$, respectivamente (34). Conviene anotar que esas dosis bajas de SDX-PIR, en comparación con la estándar, parecen seleccionar cepas de $P$. falciparum resistentes a la combinación, según observó el mismo grupo de investigadores en esos mismos niños (35). De acuerdo con Spencer et al. (1986) (36), en Malindi, Kenia, los aislamientos de $P$. falciparum que responden adecuadamente in vivo a la PIR también son muy sensibles in vitro, mientras que los resistentes in vivo también lo son in vitro: los primeros tuvieron una CIM igual a o menor de $100 \mathrm{nmol} / \mathrm{L}$, comparada con más de $299 \mathrm{nmol} / \mathrm{L}$ en los resistentes.

Por último, debe anotarse que la ausencia de una diferencia estadísticamente significativa entre los promedios o las medianas de SDX y PIR de los grupos de respuesta terapéutica podría deberse en parte al pequeño tamaño del grupo que no respondió

CUADRO 3. Valores estadísticos de las concentraciones sanguíneas de sulfadoxina (SDX) y pirimetamina (PIR) según la respuesta terapéutica

\begin{tabular}{|c|c|c|c|c|c|c|c|c|c|c|}
\hline & \multicolumn{5}{|c|}{ Sulfadoxina $(\mu \mathrm{molg} / \mathrm{L})$} & \multicolumn{5}{|c|}{ Pirimetamina (nmol/L) } \\
\hline & $X^{a}$ & $\mathrm{Me}^{\mathrm{b}}$ & $\mathrm{DE}^{\mathrm{c}}$ & IC95\% ${ }^{d}$ & $P(\mathrm{~K}-\mathrm{W})^{\mathrm{e}}$ & $x$ & $\mathrm{Me}$ & $\mathrm{DE}$ & IC95\% & $P(\mathrm{~K}-\mathrm{W})$ \\
\hline \multicolumn{11}{|l|}{ Tipo de respuesta terapéutica } \\
\hline Respuesta adecuada $(n=58)$ & 135,0 & 136,6 & 61,9 & $(73,2-196,9)$ & 0,130 & 863,7 & 848,4 & 344,8 & $(518,7-1208,5)$ & \\
\hline \multicolumn{11}{|l|}{ Tipo de respuesta } \\
\hline Fracaso temprano $(n=11)$ & 126,3 & 105,1 & 74,4 & $(76,4-176,3)$ & & 700,0 & 679,7 & 372,3 & $(433,7-966,5)$ & \\
\hline
\end{tabular}

a $X$, promedio de las concentraciones sanguíneas de SDX y PIR.

${ }^{\mathrm{b}} \mathrm{Me}$, mediana de las concentraciones sanguíneas de SDX y PIR

c DE, desviación estándar.

d IC95\%, Intervalo de confianza del 95\%.

e $P(\mathrm{~K}-\mathrm{W})$, valor $P$ asociado a la prueba de Kruskal y Wallis. 
CUADRO 4. Concentraciones sanguíneas de sulfadoxina (SDX) ( $\mu \mathrm{molg} / \mathrm{L}$ ) y pirimetamina (PIR) (nmol/L) en el día cero y en el día del fracaso terapéutico

\begin{tabular}{|c|c|c|c|c|c|c|c|c|}
\hline \multirow[b]{2}{*}{ Fármaco } & \multicolumn{4}{|c|}{ Fracaso terapéutico temprano $(\mathrm{FTP})^{\mathrm{a}}$} & \multicolumn{4}{|c|}{ Fracaso terapéutico tardío $(\mathrm{FTT})^{\mathrm{b}}$} \\
\hline & $M e^{c}$ & $\mathrm{DE}^{\mathrm{d}}$ & Mínimo & Máximo & $\mathrm{Me}$ & $\mathrm{DE}$ & Mínimo & Máximo \\
\hline SDX, día cero & 126,3 & 74,4 & 15,5 & 234,6 & 80,9 & 54,5 & 459,2 & 161,5 \\
\hline SDX, día de falla & 120,9 & 49,3 & 15,5 & 176,6 & 29,0 & 15,1 & 295,1 & 45,8 \\
\hline PIR, día cero & 721,4 & 438,7 & 184,2 & 1525,5 & 1001,6 & 444,3 & 459,2 & 1524,3 \\
\hline PIR, día de falla & 620,0 & 491,8 & 225,2 & 1623,6 & 647,8 & 369,9 & 295,1 & 1158,0 \\
\hline
\end{tabular}

a FTP: prueba de Wilcoxon; $P=0,735 \mathrm{SDX} ; P=0,866 \mathrm{PIR}$.

${ }^{b} \mathrm{FTT}$ : prueba de Wilcoxon; $P=0,063 \mathrm{SDX} ; P=0,465 \mathrm{PIR}$.

${ }^{\mathrm{C}} \mathrm{ME}=$ mediana.

${ }^{\mathrm{d}} \mathrm{DE}=$ desviación estándar.

(18 sujetos: 11 con fracaso temprano y 7 con fracaso tardío). No deja de llamar la atención que 136,6 $\mu \mathrm{mol} / \mathrm{L}$ de SDX (grupo con buena respuesta) no difieran de 103,4 $\mu \mathrm{mol} / \mathrm{L}$ (fracaso) ni de $75,7 \mu \mathrm{mol} / \mathrm{L}$ (fracaso tardío). En efecto, si se conservan los mismos pro- medios y varianzas y el tamaño de este grupo se incrementa a 40 personas, la diferencia entre los valores ya es significativa. Lo mismo sucede al comparar tres grupos (respuesta clínica adecuada, fracaso temprano, fracaso tardío): al disponer de 20 personas en cada grupo, la diferencia se vuelve significativa. Por este motivo, se trata de un tema que requiere aun mayor investigación.

Agradecimientos. El programa de control de vectores de la Dirección Seccional de Salud de Antioquia de la Gobernación de Antioquia (Colombia) financió este proyecto. Los autores desean agradecer a Armando Galeano, Director de Salud Pública de Antioquia (Colombia); a las bacteriólogas Tania Álvarez y Alexandra Ríos y a los médicos Patricia Rocha y Juan Gabriel Piñeros, integrantes del Grupo Malaria, la evaluación de la respuesta terapéutica y la obtención de las muestras. Los autores también agradecen al personal de los hospitales de ambos municipios, y especialmente a los pacientes de Turbo y Zaragoza, su participación en este estudio.

\section{REFERENCIAS}

1. Tracy JW, Webster LT Jr. Drugs used in the chemotherapy of protozoal infections. En: Hardman JG, Limbird LE, eds. Goodman \& Gilman. The Pharmacological Basis of Therapeutics. 10th ed. New York: McGraw-Hill; 2001. Pp. 1080-2.

2. Mandell G, Bennett JE, Dolin R, eds. Mandell, Douglas and Bennett. Principles and Practice of Infectious Diseases. 5th ed. Vol 1. Philadelphia: Churchill Livingstone; 2000.

3. Petri WA Jr. Antimicrobial agents. Sulfonamides, trimetoprim-sulfamethoxazole, quinolones and agents for urinary infections. En: Hardman JG, Limbird LE, eds. Goodman \& Gilman. The Pharmacological Basis of Therapeutics, $10^{\underline{a}}$ ed. New York: McGraw-Hill; 2001;1171-6.

4. Heizmann P, Fernex M, Leimer R, ReberLiske R, Stohler H. Plasmodium falciparum resistant to mefloquine plus sulfadoxine/ pyrimethamine. Lancet. 1986;1(8477):377.

5. Malin AS, Hall AP. Falciparum malaria resistant to quinine and pyrimethaminesulfadoxine successfully treated with mefloquine. Br Med J. 1990;300(6733):1175.

6. Ronn AM, Msangeni HA, Mhina J, Wernsdorfer WH, Bygbjerg IC. High level of resistance of Plasmodium falciparum to sulfadoxinepyrimethamine in children in Tanzania. Trans R Soc Trop Med Hyg. 1996;90(2):179-81.

7. Onyiorah E, van Hensbroek MB, Jah MS, Greenwood B. Early clinical failures after pyrimethamine-sulfadoxine treatment of uncomplicated falciparum malaria. Trans R Soc Trop Med Hyg. 1990;90(3):307-8.

8. Sowunmi A, Oduola AM, Ogundahunsi OA, Salako LA. Comparative efficacy of chloroquine plus chlorpheniramine and pyrimethamine/sulfadoxine in acute uncom- plicated falciparum malaria in Nigerian children. Trans R Soc Trop Med Hyg. 1998;92(1): 77-81.

9. Van Dillen J, Custers M, Wensink A, Wouters B, van Voorthuizen T, Voorn W, et al. A comparison of amodiaquine and sulfadoxinepyrimethamine as first-line treatment of falciparum malaria in Kenya: 1. Trans R Soc Trop Med Hyg. 1999;93(2):185-8.

10. Deloron P, Mayombo J, Le Cardinal A, MezuiMe-Ndong J, Bruzi-Baert C, Lekoulou F, Elissa N. Sulfadoxine-pyrimethamine for the treatment of Plasmodium falciparum malaria in Gabonese children. Trans R Soc Trop Med Hyg. 2000;94(2):188-90.

11. Nwanyanwu OC, Ziba C, MacHeso A, Kazembe P. Efficacy of sulphadoxinepyrimethamine for acute uncomplicated malaria due to Plasmodium falciparum in Malawian children under five years old. Trop Med Int Health. 2000;5(5):355-8.

12. Von Seidlein L, Milligan P, Pinder M, Bojang $\mathrm{K}$, Anyalebechi C, Gosling R, et al. Efficacy of artesunate plus pyrimethamine-sulphadoxine for uncomplicated malaria in Gambian children: a double-blind, randomised, controlled trial. Lancet. 2000;355(9201):352-7. Erratum in: Lancet. 2000;355(9220):2080.

13. Staedke SG, Kamya MR, Dorsey G, Gasasira A, Ndeezi G, Charlebois ED, et al. Amodiaquine, sulfadoxine/pyrimethamine, and combination therapy for treatment of uncomplicated falciparum malaria in Kampala, Uganda: a randomised trial. Lancet. 2001; 358(9279):368-74.

14. Tjitra E, Suprianto S, Currie BJ, Morris PS, Saunders JR, Anstey NM. Therapy of uncomplicated falciparum malaria: a randomized trial comparing artesunate plus sulfadoxine-pyrimethamine versus sulfadoxinepyrimethamine alone in Irian Jaya, Indonesia. Am J Trop Med Hyg. 2001;65(4): 309-17.

15. Blair S, Lacharme L, Carmona J, Tobón A. Resistencia del Plasmodium falciparum a los antimaláricos en Urabá y Bajo Cauca Antioqueño, 1998. Rev Epidemiol Antioquia. 1999;24(3-4): 207-15.

16. Blair S. Resistencia a los antimaláricos. En: Carmona-Fonseca J. Tópicos de infectología. Medellín: Universidad de Antioquia, 1999; 81-91.

17. Blair S, Lacharme-Lora L, Carmona-Fonseca J. Resistance of Plasmodium falciparum to antimalarial drugs in Zaragoza (Antioquia, Colombia), 1998. Mem Inst Oswaldo Cruz. (Rio de Janeiro) 2002;97(3):401-6.

18. Blair S, Lacharme L, Carmona J, Tobón A. Resistencia del Plasmodium falciparum a tres fármacos antimaláricos en Turbo (Antioquia, Colombia), 1998. Rev Panam Salud Publica. 2001;9(1):23-9.

19. Espinal CA, Uribe LM, Eslava A, Rodríguez ME. Resistencia del $P$. falciparum a la combinación sulfadoxina-pirimetamina. Descripción de los primeros tres casos en Colombia. Biomedica. 1981;1(4):213-7.

20. Botero D, Restrepo M, Montoya A. Prospective double-blind trial of two different doses of mefloquine plus pyrimethaminesulfadoxine compared with pyrimethaminesulfadoxine alone in the treatment of $P$. falciparum malaria. Bull World Health Organ. 1985;63:731-7.

21. Soto J, Martínez R, Fonschiffrey G, Dember J. Efficacy of Fansidar for acute uncomplicated malaria due to Plasmodium falciparum in Colombia. Trans R Soc Trop Med Hyg. 1995; 89(3):306. 
22. Blair S. Resistencia de P. falciparum a drogas en Colombia. Biomédica. 1986;6(3-4):95-100.

23. Osorio LE, Giraldo LE, Grajales LF, Arriaga AL, Andrade AL, Ruebush TK 2nd, et al. Assessment of therapeutic response of Plasmodium falciparum to chloroquine and sulfadoxinepyrimethamine in an area of low malaria transmission in Colombia. Am J Trop Med Hyg. 1999;61(6):968-72.

24. Osorio LE, Giraldo LE, Grajales LF, Barat LM, Córdoba F, Arriaga AL, et al. Evaluación in vivo de la resistencia de Plasmodium falciparum a cloroquina y sulfa/pirimetamina en Quibdó, Chocó. Biomedica. 1997; 17(supl. 2): 201-2.

25. López YL, Arroyabe A, Salazar A. Evaluación de la resistencia in vivo a los medicamentos antimaláricos. El Bagre, Antioquia, 1998. Rev Epidemiol Antioquia. 1999;24(3-4):181-94.

26. Ministerio de Salud de Colombia, Dirección General de Salud Pública, Grupo de Vigilancia en Salud Pública. Casos y tasa de incidencia de malaria por municipio según especie. Santa Fe de Bogotá: Minsalud; 2001.

27. Sierra ML, Vélez LM, Castañeda AM, Galeano LA, Molina AL, Tabares Z, et al. Diagnóstico de la situación de salud en Antioquia. Rev Epidemiol Antioquia. 2000;25(1-3):129-32.

28. López-Antuñano FJ, Schmunis G, eds. Diagnóstico de malaria. Washington, D.C.: Organi- zación Panamericana de la Salud, 1988. (Publicación Científica 512).

29. Organización Panamericana de la Salud. Evaluación de la eficacia terapéutica de los medicamentos para el tratamiento del paludismo por Plasmodium falciparum sin complicaciones en las Américas. Washington, D.C.: OPS; 1998. (Documento OPS/HCP/HCT/ 113/98).

30. Colombia, Ministerio de Salud. Guía de atención clínica para el diagnóstico y tratamiento de la malaria. Santa Fe de Bogotá; Minsalud; 1999.

31. Green M, Mount D, Netty H. Highperfomance liquid cromatographic assay for the simultaneous determination of sulfadoxine and pyrimethamine from whole blood dried onto filter paper. J Chromatogr B Analyt Technol Biomed Life Sci. 2002;767(1): 159-62

32. Weidekamm E, Plozza-Nottebrock H, Forgo I, Dubach UC. Plasma concentrations in pyrimethamine and sulfadoxine and evaluation of pharmacokinetic data by computerized curve fitting. Bull World Health Organ. 1982;60(1): 115-22.

33. Lell B, Lehman LG, Schmidt-Ott JR, Sturchler D, Handschin J, Kremsner PG. Malaria chemotherapy trial at a minimal effective dose of mefloquine/sulfadoxine/ pyrimethamine compared with equivalent doses of sulfadoxine/pyrimethamine or mefloquine alone. Am J Trop Med Hyg. 1998; 58(5):619-24.

34. Hellgren U, Kihamia CM, Bergqvist Y, Lebbad M, Premji Z, Rombo L. Standard and reduced doses of sulfadoxine-pyrimethamine for treatment of Plasmodium falciparum in Tanzania, with determination of drug concentrations and susceptibility in vitro. Trans R Soc Trop Med Hyg. 1990;84(4):469-72.

35. Kun JF, Lehman LG, Lell B, Schmidt-Ott R, Kremsner PG. Low-dose treatment with sulfadoxine/pyrimethamine combinations selects for drug-resistant Plasmodium falciparum strains. Antimicrob Agents Chemother. 1999;43(9):2205-8.

36. Spencer HC, Watkins WW, Sixsmith DG, Koech DK. Response of Plasmodium falciparum to dihydrofolate reductase inhibitors in Malindi, Kenya. Trans R Soc Trop Med Hyg. 1986;80(2):201-13.

Manuscrito recibido el 2 de septiembre de 2004. Aceptado para publicación, tras revisión, el 29 de marzo de 2005.
ABSTRACT

Blood levels of sulfadoxine and pyrimethamine, according to the malaria-treatment response, in two municipalities of Antioquia, Colombia

\footnotetext{
Keywords
}

Problem. There has been a constant increase in the level of therapeutic failure of the sulfadoxine-pyrimethamine (SP) combination for treating uncomplicated Plasmodium falciparum malaria.

Objective. To use high-performance liquid chromatography to quantify blood levels of SP in patients with good clinical response and in patients who did not respond to treatment.

Methods. This experimental study was carried out in 2002 in Turbo and Zaragoza, two municipalities in the department of Antioquia in Colombia. There were 79 patients (45 in Turbo and 34 in Zaragoza), including both men and women, who ranged in age from 1 year to 60 years. All the patients had uncomplicated Plasmodium falciparum malaria, with a parasite density of 500 to 50000 parasites $/ \mu \mathrm{L}$. The patients were each randomly assigned to a treatment group. The treatment groups were not blinded; the physician who provided the medication also evaluated the therapeutic response. The treatment consisted of a single combination dose of sulfadoxine $(25 \mathrm{mg} / \mathrm{kg})$ and pyrimethamine $(1.25 \mathrm{mg} / \mathrm{kg})$ in tablets $(500 \mathrm{mg}$ of sulfadoxine and $25 \mathrm{mg}$ of pyrimethamine). Clinical-parasitological follow-up was carried out for 21 days. Blood levels of sulfadoxine and pyrimethamine were measured two hours after the treatment was given and also the day of treatment failure, if that occurred.

Results. Two hours after the treatment was given, the median blood level of sulfadoxine was $136.6 \mu \mathrm{mol} / \mathrm{L}$ in the patients who later showed a good clinical response, and it was $103.4 \mu \mathrm{mol} / \mathrm{L}$ among those who did not respond to treatment $(P=0.13)$. The medians for pyrimethamine were $848.4 \mathrm{nmol} / \mathrm{L}$ in patients with a good clinical response and $786.1 \mathrm{nmol} / \mathrm{L}$ in patients with treatment failure $(P=0.40)$. There were no significant differences in drug levels between the early-failure cases and the latefailure cases. The linear correlation between the blood levels of sulfadoxine and pyrimethamine was close to zero $(r=0.13)$.

Conclusions. Between 1998 and 2002, treatment failure with the SP combination increased from $13 \%$ to $22 \%$ in Turbo, and from $9 \%$ to $26 \%$ in Zaragoza. The lack of response in 2002 could not be explained by lower blood levels of the medications.

Malaria, falciparum; antimalarials; sulfadoxine; pyrimethamine; chromatography, high pressure liquid. 